\title{
Microarray analysis of a salamander hopeful monster reveals transcriptional signatures of paedomorphic brain development
}

\author{
Robert B Page ${ }^{+1}$, Meredith A Boley ${ }^{\dagger 1}$, Jeramiah J Smith ${ }^{1,2}$, Srikrishna Putta ${ }^{1}$ and Stephen R Voss ${ }^{* 1}$
}

\begin{abstract}
Background: The Mexican axolotl (Ambystoma mexicanum) is considered a hopeful monster because it exhibits an adaptive and derived mode of development - paedomorphosis - that has evolved rapidly and independently among tiger salamanders. Unlike related tiger salamanders that undergo metamorphosis, axolotls retain larval morphological traits into adulthood and thus present an adult body plan that differs dramatically from the ancestral (metamorphic) form. The basis of paedomorphic development was investigated by comparing temporal patterns of gene transcription between axolotl and tiger salamander larvae (Ambystoma tigrinum tigrinum) that typically undergo a metamorphosis.
\end{abstract}

Results: Transcript abundances from whole brain and pituitary were estimated via microarray analysis on four different days post hatching $(42,56,70,84 \mathrm{dph})$ and regression modeling was used to independently identify genes that were differentially expressed as a function of time in both species. Collectively, more differentially expressed genes (DEGs) were identified as unique to the axolotl $(n=76)$ and tiger salamander $(n=292)$ than were identified as shared $(n=108)$. All but two of the shared DEGs exhibited the same temporal pattern of expression and the unique genes tended to show greater changes later in the larval period when tiger salamander larvae were undergoing anatomical metamorphosis. A second, complementary analysis that directly compared the expression of 1320 genes between the species identified 409 genes that differed as a function of species or the interaction between time and species. Of these 409 DEGs, 84\% exhibited higher abundances in tiger salamander larvae at all sampling times.

Conclusions: Many of the unique tiger salamander transcriptional responses are probably associated with metamorphic biological processes. However, the axolotl also showed unique patterns of transcription early in development. In particular, the axolotl showed a genome-wide reduction in mRNA abundance across loci, including genes that regulate hypothalamic-pituitary activities. This suggests that an axolotls failure to undergo anatomical metamorphosis late in the larval period is indirectly associated with a mechanism(s) that acts earlier in development to broadly program transcription. The axolotl hopeful monster provides a model to identify mechanisms of early brain development that proximally and ultimately affect the expression of adult phenotypes.

\section{Background}

Darwin [1] proposed that evolution by natural selection is a gradual process that results in continuous phenotypic variation among species. However, there are many examples where discontinuous phenotypes are observed among related species and thus appear to evolve rapidly.

\footnotetext{
*Correspondence: srvoss@uky.edu

1 Department of Biology and Spinal Cord and Brain Injury Research Center,

University of Kentucky, Lexington, KY 40506, USA

+ Contributed equally

Full list of author information is available at the end of the article
}

That evolution could suddenly "leap forward" led to extensions of Darwin's theory to account for the rapid origin of novel phenotypes. One very old idea is that novel and dramatically different phenotypes originate via saltational evolution from mutations of genes that regulate key developmental or physiological processes during ontogeny. In particular, Goldschmidt [2] proposed that mutations occasionally yield individuals within populations that deviate radically from the norm and referred to such individuals as "hopeful monsters". If the novel phenotypes of hopeful monsters arise under the right envi- 
ronmental circumstances, they may become fixed, and the population will found a new species. While this idea was discounted during the Modern Synthesis [3], aspects of the hopeful monster hypothesis have been substantiated in recent years. For example, it is clear that dramatic changes in phenotype can occur from few mutations of key developmental genes and phenotypic differences among species often map to relatively few genetic factors [4-8]. These findings are motivating renewed interest in the study of hopeful monsters and the perspectives they can provide about the evolution of development $[9,10]$. In contrast to mutants that are created in the lab, hopeful monsters have been shaped by natural selection and are therefore more likely to reveal mechanisms of adaptive evolution.

At least three lines of evidence led Goldschmidt [2] to cite the Mexican axolotl (Ambystoma mexicanum) as one of the original hopeful monsters. First, the axolotl follows a different ontogeny from other closely related tiger salamanders. Whereas some tiger salamanders undergo an obligatory metamorphosis during ontogeny that allows for a transition from an aquatic habitat to a more terrestrial habitat, the axolotl has a non-metamorphic life cycle that is often referred to as paedomorphic [11]. This extreme example of discontinuous phenotypic variation supports a model of evolution by heterochrony: larval morphological traits of ancestral metamorphic forms are observed in the adult stages of derived paedomorphic forms. In the minds of early evolutionary biologists, these patterns were so clearly supportive of heterochrony that the Mexican axolotl became the exemplar of evolution by neoteny $[12,13]$. The second reason Goldschmidt cited the axolotl was physiological - Huxley [14] had shown that a single molecule - thyroid hormone (TH) - was capable of rescuing metamorphosis in the axolotl. Thus, the axolotl seemed to be an example of evolution waiting around for the right macromutation to happen - simply block a single physiological step in TH regulation and a novel form is originated. The third reason Goldschmidt cited the axolotl was ecological. Previous researchers had noted that the axolotl was endemic to the high quality, permanent aquatic habitats of Xochimilco, which is near present day Mexico City [15]. The evolution of paedomorphosis seemingly allowed the axolotl to exploit an empty niche in an environment that was devoid of predators.

Since Goldschmidt, the axolotl has remained a quintessential hopeful monster [16]. Speculation that the paedomorphic condition of the axolotl could have a simple mechanistic basis was supported when a quantitative trait locus (QTL) was identified for the segregation of metamorphic and paedomorphic phenotypes in interspecific crosses [8,17-20]. Previous physiological studies had also established that axolotls do not produce a sufficient titer of thyroid hormone during larval development to initiate anatomical metamorphosis [[21,22] reviewed in [23-25]]. The evolution of axolotl hypothyroidism is thought to be associated with a mechanism that affects the development and/or function of neuroendocrine axes that regulate the release of thyroid hormone from the thyroid glands [11,26,27]. Conceivably, this mechanism could function during early stages of development or it could function later in the larval period when metamorphosis is initiated. Regardless, whether paedomorphic and metamorphic larvae show similar or different patterns of neurological development and function has not been previously investigated.

In this study, microarray analysis was used to investigate transcription within whole brains (including the pituitary) of the paedomorphic axolotl and a closely related metamorphic species (A. tigrinum tigrinum). The primary objective was to identify patterns of gene expression during early ontogeny that could provide new mechanistic insights about paedomorphic and metamorphic modes of development. Transcripts were sampled from both species at four chronologically matched times post hatching to obtain temporal profiles of gene expression during the early larval period and during early stages of morphological metamorphosis in A. t. tigrinum. Hundreds of genes showed different or unique patterns of expression between the species, many of which were initiated very early in the larval period and prior to the onset of morphological metamorphosis. The results suggest considerable potential for transcriptional divergence between closely related vertebrate species and highlight the tiger salamander/axolotl model system for examining mechanisms in the developing brain that determine adult phenotypic outcomes.

\section{Results}

\section{Larval growth and metamorphosis}

Under normal growth conditions, larvae of metamorphic and paedomorphic species of Ambystoma increase in size but only larvae of metamorphic species show changes in morphology (bulging eyes, changes in head shape, reduction of tailfins and gills) that are indicative of anatomical metamorphosis. In this experiment, tiger larvae were larger than axolotls at $28 \mathrm{dph}$ and exhibited higher growth rates early in the larval period (Figure 1). As development proceeded, tiger salamander growth rates decelerated while axolotl growth rates remained constant. None of the tiger larvae showed changes in morphology suggestive of initiation of metamorphosis at the three earliest time points $(28,42,56 \mathrm{dph})$. However at 70 dph, 23\% $(n=30)$ of tiger larvae showed bulging eyes and subtle changes in head morphology. By $84 \mathrm{dph}$, all tiger larvae were undergoing anatomical metamorphosis and $20 \%(n=30)$ of these had rudiments of gills that were less 


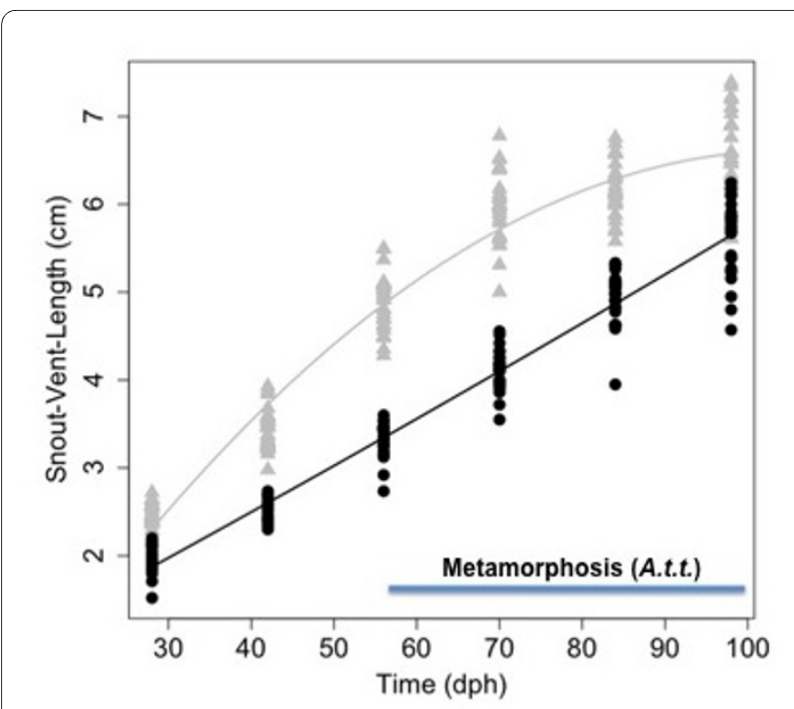

Figure 1 Growth of larval axolotls and tiger salamanders. Data points are snout vent length (SVL) measured for individuals at the time of brain tissue collection. Lines were estimated by fitting a general linear model $\left(R^{2}=0.957\right)$ to SVL data from both species. All terms of the model were significant $(p<0.05$; see methods). Axolotl larvae $=$ black circles, tiger salamander larvae = gray triangles. The blue bar shows the time period where anatomical changes consistent with metamorphosis were observed in tiger salamander larvae (A.t.t.).

than $1 \mathrm{~mm}$ in length. These larvae, and all larvae that were examined at $98 \mathrm{dph}(n=30)$, were considered metamorphs. Thus, some tiger salamanders initiated anatomical metamorphosis between 56 and $70 \mathrm{dph}$ and most larvae completed metamorphosis between 84 and $98 \mathrm{dph}$.

\section{Differentially expressed genes identified independently from axolotls and tiger salamanders}

A custom Affymetrix GeneChip [see [28-30]] was used to estimate mRNA abundances at four chronologically matched times $(42,56,70$, and $84 \mathrm{dph})$ to obtain temporal profiles of gene expression for tiger salamander and axolotl larvae. Statistical and fold-change criteria were then used to identify genes that were differentially expressed within each of the species. Thus, these analyses took the conservative approach of independently identifying genes from each species that were differentially expressed as a function of time and comparing the list of differentially expressed genes (DEGs) from tiger salamander with the list of DEGs identified from axolotl. This approach is conservative because it reduces the risk of identifying false positive expression differences between species that are caused by heterologous hybridization [31]. More than twice as many DEGs were identified from tiger salamander than axolotl larvae ( $n=400$ vs 184 ) (Figure 2 ). There was considerable overlap between the two species as more than half $(n=108)$ of the genes identified from axolotls were also identified from tiger salamander larvae.

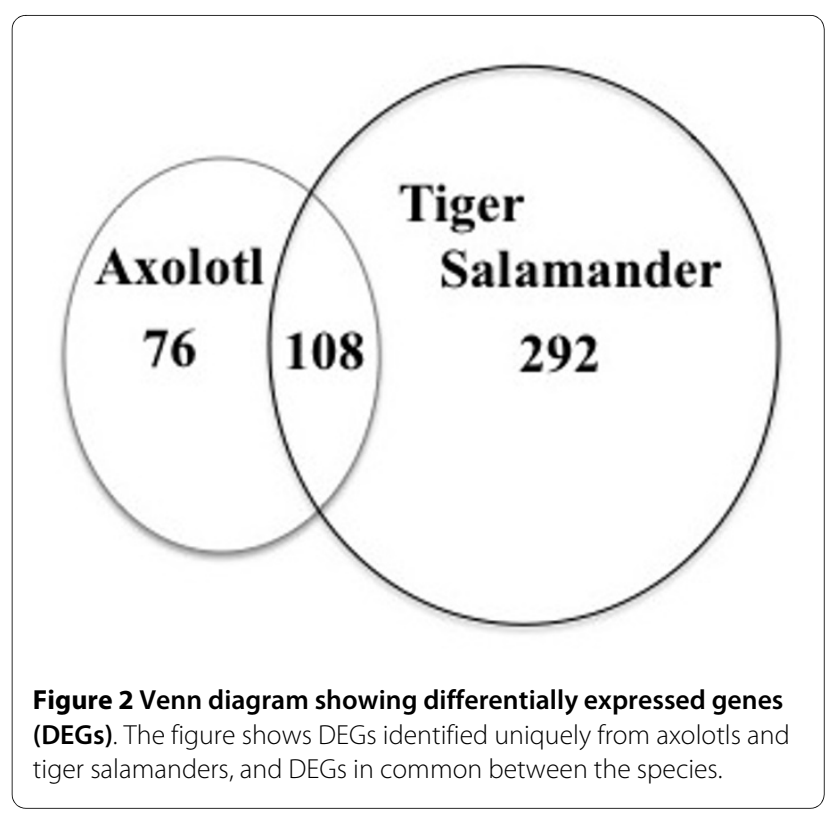

The remaining DEGs changed uniquely in only one species. Thus, many shared and unique DEGs were identified, with more unique DEGs identified from tiger salamander larvae.

Of the 108 DEGs identified from both species, 60\% exhibited the same regression profile and $99 \%$ exhibited the same generalized direction of differential expression (i.e., up versus down-regulation; Additional File 1). Of the 76 DEGs uniquely identified from axolotls (Additional File 2), 55\% showed increasing mRNA abundances (LU, QLVU, or QLCU) during the larval period while 45\% showed decreasing abundances (LD, QLVD, or QLCD) (see Figure 3 for acronym definitions). The opposite pattern was observed for the 332 unique tiger salamander DEGs with 55\% showing decreasing mRNA abundances, $43 \%$ showing increasing abundances, and $1 \%$ showing a pattern of transient increase $(\mathrm{QC})$. Only a small percentage $(\sim 10 \%)$ of shared and unique DEGs exhibited $\geq 1.5$ fold difference in mRNA abundance between the earliest time points ( 42 and $56 \mathrm{dph}$ ). However, between the latest time points (70 and $84 \mathrm{dph}$ ), $\sim 32 \%$ of the shared DEGs, $64 \%$ of unique axolotl DEGs, and $61 \%$ of unique tiger salamander DEGs showed $a \geq 1.5$ fold difference. Thus, abundances of uniquely expressed transcripts tended to increase throughout larval development, showing the largest fold differences at later larval stages.

The DEGs identified independently from both species that showed significant sequence identity to a human RefSeq protein were assumed to be salamander-human orthologs and were annotated with biological process information from the Gene Ontology (GO) database. Many of the same GO terms were represented among the three DEG lists - the DEGs that were expressed in common between the species (i.e. shared list) and the separate 


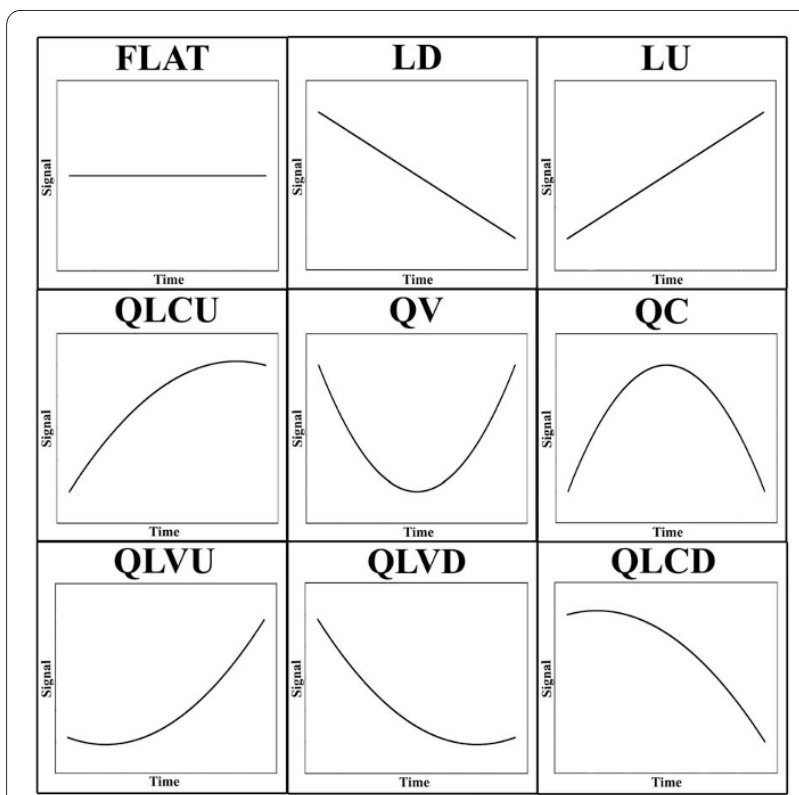

Figure 3 Regression patterns of differentially expressed genes identified from axolotls and tiger salamanders. $L D=$ linear down, $\mathrm{QLCD}=$ quadratic linear concave down, QLVD = quadratic linear convex down, $\mathrm{QV}=$ quadratic convex, $\mathrm{LU}=$ linear $\mathrm{up}, \mathrm{QLVU}=$ quadratic linear convex up, QLCU = quadratic linear concave up, and QC = quadratic concave.

lists of DEGs that were identified uniquely from each species (i.e. axolotl or tiger salamander). The commonly expressed DEGs were statistically associated with 27 terms, and many of these genes are predicted to function in cell cycle processes (Figure 4). Nearly all of the DEGs that annotated to cell cycle terms exhibited decreasing mRNA abundances during larval development in both species, as did six of seven annotated transcription factors. For example, two biomarkers of neural development and differentiation (sox3, $m s \times 1$ ) were both categorized as LD in axolotl and tiger salamander. Conversely, the majority of DEGs associated with system development were up regulated in both species. These results suggest that some aspects of neural development and function are similarly regulated between axolotl and tiger salamander larvae. This also seems to be true for some but not all hemoglobin loci, and genes that function in stress and immunological pathways.

Relatively few uniquely expressed axolotl genes were identified overall $(\mathrm{N}=76)$ and thus only 3 broad GO terms were identified as statistically enriched (Figure 4): regulation of cellular process $(n=21, p=0.004)$, regulation of biological process $(n=21, p=0.011)$, and biological regulation $(n=22, p=0.015)$. The unique axolotl genes are predicted to function in some but not all of the biological processes observed for the shared DEG list (Additional File 2). For example, six genes that function in apoptosis (srpbp1, anax1, anax5, mtch1, gstp1, pim1) were uniquely identified for axolotls. DEGs known to be associated with vertebrate brain development were identified, including genes that code for extracellular matrix constituents and cell adhesion (e.g., mmp1, dcn, colla1, $d p t$, lgals4). Also, several biomarkers of mammalian brain pathologies were uniquely up regulated in axolotls (ctss, ogn, cd69). These and other uniquely expressed genes may be associated with the axolotl's paedomorphic mode of development.

The larger list of unique DEGs from tiger larvae yielded more biological process annotations and were statistically associated with 23 GO terms (Figure 4)(Additional File 3). As was observed for the shared gene list, the cell cycle GO term was significantly enriched and these genes showed decreasing mRNA abundances during larval development. However, several GO terms were identified that were not represented in the shared or unique axolotl list, including biological processes associated with chromatin organization and biogenesis. For example, $\operatorname{lm} x 1 b$ showed a pattern of decreasing transcript abundance, as did several other genes that function in chromatin organization, modification, and gene silencing (e.g. dnmt1, baz1b, baz1a, smarca5, hist1h1b, hist1hbj, hist2h2ac). It is possible that some of these unique DEGs are associated with the maturation of brain regions that orchestrate metamorphic events. For example, several genes that function to regulate the secretion of hypothalamic, pituitary, and interrenal hormones were uniquely expressed in tiger salamander larvae, including $n r 3 c 2$, prl, and sstr5. In addition to these genes, pomc and crhrl exhibited higher expression levels in tiger salamander larvae (see real-time PCR results below). Thus, the microarray analysis identified expression differences between axolotl and tiger larvae that may correlate with HPI axis regulation and function.

\section{Direct comparison of transcription between axolotl and tiger salamander}

To complement the statistical analyses described above, a subset of Affymetrix probesets were identified that could be used to reliably compare transcript abundance estimates directly between the species. Othologous genes from axolotl and tiger salamander were aligned to identify 1320 Affymetrix probe-sets with zero mismatches between the species. Ten of these probesets were identical to other probesets. Of the remaining non-redundant probesets, $31 \%(n=409)$ registered statistically distinct expression profiles between the species. This analysis identified some of the unique, temporally regulated DEGs described above, and additionally, genes that exhibited flat regression profiles that differed significantly in elevation between the species. Indeed, $84 \%(n=343)$ of the genes identified from this analysis showed higher expression levels throughout the larval period in tiger salaman- 
Response to endogenous stimulus

DNA repair

Chromosome organization and biogenesis

Microtubule-based process

Interphase

Anion transport

Second-messenger-mediated signaling

Phosphoinositide-mediated signaling

Response to other organism

Nucleosome assembly

Chromatin assembly

Spindle organization and biogenesis

Base-excision repair

Glycolipid metabolic process

DNA damage checkpoint

Adaptive immune response

Oxygen transport

Mitotic cell cycle checkpoint

DNA duplex unwinding

Regulation of mitosis

Regulation of cyclin-dependent protein kinase

Response to wounding

DNA replication initiation

Cell cycle checkpoint

Response to external stimulus

System development

Cell proliferation

Response to stimulus

Mitotic sister chromatid segregation

DNA-dependent DNA replication

DNA replication

Cell division

Mitosis

Mitotic cell cycle

M phase

DNA metabolic process

Response to stress

Cell cycle

Regulation of cellular process

Regulation of biological process

Biological regulation

\section{$\begin{array}{llllll}\mathbf{0} & \mathbf{1 0} & \mathbf{2 0} & \mathbf{3 0} & \mathbf{4 0} & \mathbf{5 0}\end{array}$ \\ Number of Genes}

Figure 4 Gene ontology (GO) terms identified as significantly over-represented. The figure shows $\mathrm{GO}$ terms for differentially expressed genes (DEGs) identified uniquely from axolotls (black bars) and tiger salamanders (red bars), and GO terms for DEGs that were identified in common between the species (blue bars). 

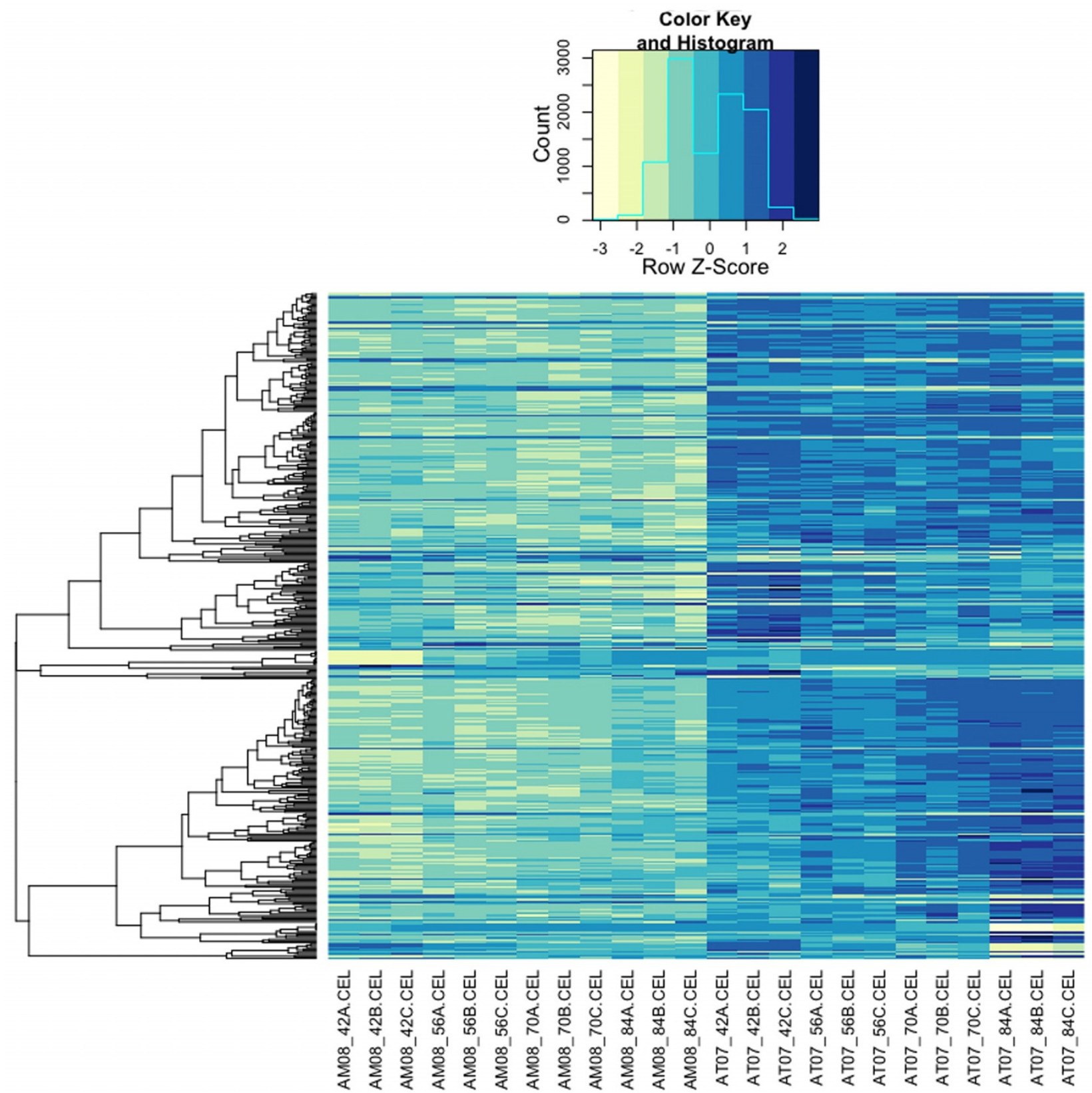

Figure 5 Heat map of differentially expressed genes. The figure shows row centered and scaled data from 419 genes identified by directly comparing transcript abundances between axolotls and tiger salamanders. The columns correspond to individual GeneChips with the prefixes AM and AT denoting axolotl GeneChips and tiger salamander GeneChips respectively. Time points correspond to days post hatching and are denoted by numbers followed by a letter (e.g., AM08_42C.CEL corresponds to an RNA pool derived from axolotls sampled at $42 \mathrm{dph}$ ). The dendrogram to the left was obtained via hierarchical clustering of a pair-wise distance matrix that was calculated as $|1-r|$, where $r=$ Pearson's correlation coefficient.

ders (Figure 5; Additional File 4). This list is statistically enriched with genes that function in oxygen/gas transport $(n=4, p=0.012)$, lipid transport $(n=6, p=0.035)$, and heterocycle metabolic processes $(n=7, p=0.038)$. With respect to oxygen transport, several hemoglobin genes $(h b g 1, h b e 1, h b d, h b a)$ exhibited > 10 fold differences between the species. The complete list of genes that showed higher abundances in tiger salamanders annotate to many different biological processes, including mRNA biosynthesis (med8, med31, polr $2 d$, taf8, taf12), protein translation (eef1g, eif2s1, eif3i), post-translational modification of proteins (spop, sumo1, uba2, ube2e3, ebe2i, ube2l3, ube2r2, usp2), neural function and development (aldh1a1, bambi, id3, smad5, sigmar1, $n r 3 c 2$ ), stress and 
immune response (cat, hmox1, ifi44l, gpx1, oxr1, sftpd), and hypothalamic-pituitary function $(f s t, n r 3 c 2$, tef). The relatively few genes $(n=41)$ that showed consistently higher transcript abundances in axolotl larvae also annotate to a number of different biological processes, including DNA metabolism (kpna2, rrm1, rrm2), cell cycle regulation (pim3, cdk4, cdc20), collagen metabolism (mmp1, mmp9, col3a1, col6a1, colsa1), neural development (ndrg2), and cellular metabolism (eno1, pgym, fbp2, acy3). It is unlikely that the interspecific expression differences described above are artifacts of heterologous hybridization because $>80 \%$ of the probe-sets were designed from the species (axolotl) that showed consistently lower transcriptional abundances across genes. These results further demonstrate that genes are differentially expressed between the developing brains of axolotl and tiger salamander larvae, and that mRNA abundance is on average higher for tiger salamander orthologs.

\section{Further investigation of gene expression using qPCR}

Some of the DEGs that were identified by microarray analysis suggest the HPI axis is differentially regulated between tiger salamander and axolotl larvae. For example, microarray analysis estimated higher transcript abundances for $n r 3 c 2$ in tiger salamanders, while $n r 3 c 1$ did not differ between the species. In adult mammals and presumably amphibians, glucocorticoids feedback to regulate brain development and function by binding to $n r 3 c 1$ and $n r 3 c 2$, which like thyroid hormone receptors, act as transcription factors. To verify transcript abundance estimates for these genes, and extend the analysis to two additional HPI axis genes ( $c r h r 1$, pomc), quantitative realtime reverse transcription PCR (qPCR) was used to examine mRNA expression across a broader range of time points (28 - $98 \mathrm{dph}$; Figure 6). Both $n r 3 c 1$ and $n r 3 c 2$ showed a pattern of increasing transcript abundance with maximal levels attained at $70 \mathrm{dph}$ for $n r 3 c 1$ and $84 \mathrm{dph}$ for $n r 3 c 2$; after these times, levels decreased. Whereas $n r 3 c 1$ showed the same pattern of expression in both species, $n r 3 c 2$ abundance was two fold higher in tiger salamander larvae at $84 \mathrm{dph}$. A pattern of increasing transcript abundance was also observed across the larval period for pomc while crhr1 mRNA levels remained relatively flat. For both of these genes, mRNA levels were significantly higher in tiger salamander larvae. These qPCR results corroborate the earlier microarray results and show that HPI axis genes are more highly expressed in tiger salamanders during larval development.

The microarray analysis also showed dramatic changes in hemoglobin mRNA abundances both within and between species. Presumably, these gene expression patterns reflect transcription within erythrocytes that were isolated from vasculature within the brain. The Ambystoma genome appears to encode multiple paralogs for almost all hemoglobin loci and these are expressed differently in some cases (unpublished data). For example, paralogs of $h b a$ in tiger salamander showed both increasing (SRV_00496_s_at) and decreasing (SRV_02508_x_at) patterns of mRNA abundance, while all axolotl $h b a$ paralogs showed flat or increasing patterns (Additional File 1). qPCR yielded a flatter profile for axolotl $h b a$ than was suggested by the microarray analysis, however qPCR replicated the dramatic decrease in mRNA abundance for tiger salamander $h b a$, as well as the expression patterns of three additional hemoglobin loci for both species (Figure 7). Overall, the patterns suggest that axolotls and tiger salamanders similarly up regulate some of the same hemoglobin loci $(h b d, h b a, h b g)$ during the larval period, although levels were consistently higher in tiger salamander larvae. The only locus that was similarly down regulated was $h b z$. Interestingly, axolotls maintain higher embryonic-type hemoglobin (hbe) mRNA levels than tigers throughout the larval period, a pattern that is consistent with a paedomorphic mode of development.

\section{Discussion}

This study used a functional genomics approach to detail larval brain transcription between the paedomorphic Mexican axolotl and metamorphic tiger salamander. The results show that larvae of these species have different transcriptional programs that are distinguishable in two important respects. First, although shared expression patterns were observed between the species, most of the genes that were identified as differentially expressed during the larval period showed species-specific patterns of expression. Gene expression was more similar between the species at earlier time points, with pronounced differences observed at 70 and $84 \mathrm{dph}$, which coincided with the onset of anatomical metamorphosis in a subset of the tiger salamander larvae. Second, the abundance of mRNAs tended to be higher for genes that were up regulated during tiger salamander development, relative to those that were up regulated during axolotl development. Approximately $31 \%$ of genes that could be reliably and directly compared between the species were differentially expressed and $84 \%$ of these showed higher mRNA abundances in tiger salamander larvae. Below, we discuss these primary results and explore their relationships to transcriptional programming that may correlate with metamorphic and paedomorphic modes of development.

Similar expression patterns were observed for $99 \%$ of the genes that were commonly differentially expressed in axolotl and tiger salamander larvae as a function of time. This suggests that some biological processes are regulated similarly between axolotl and tiger salamander larvae during development. For example, it is possible that some of the genes that function in the specification and proliferation of neuronal cell types are similarly expressed 

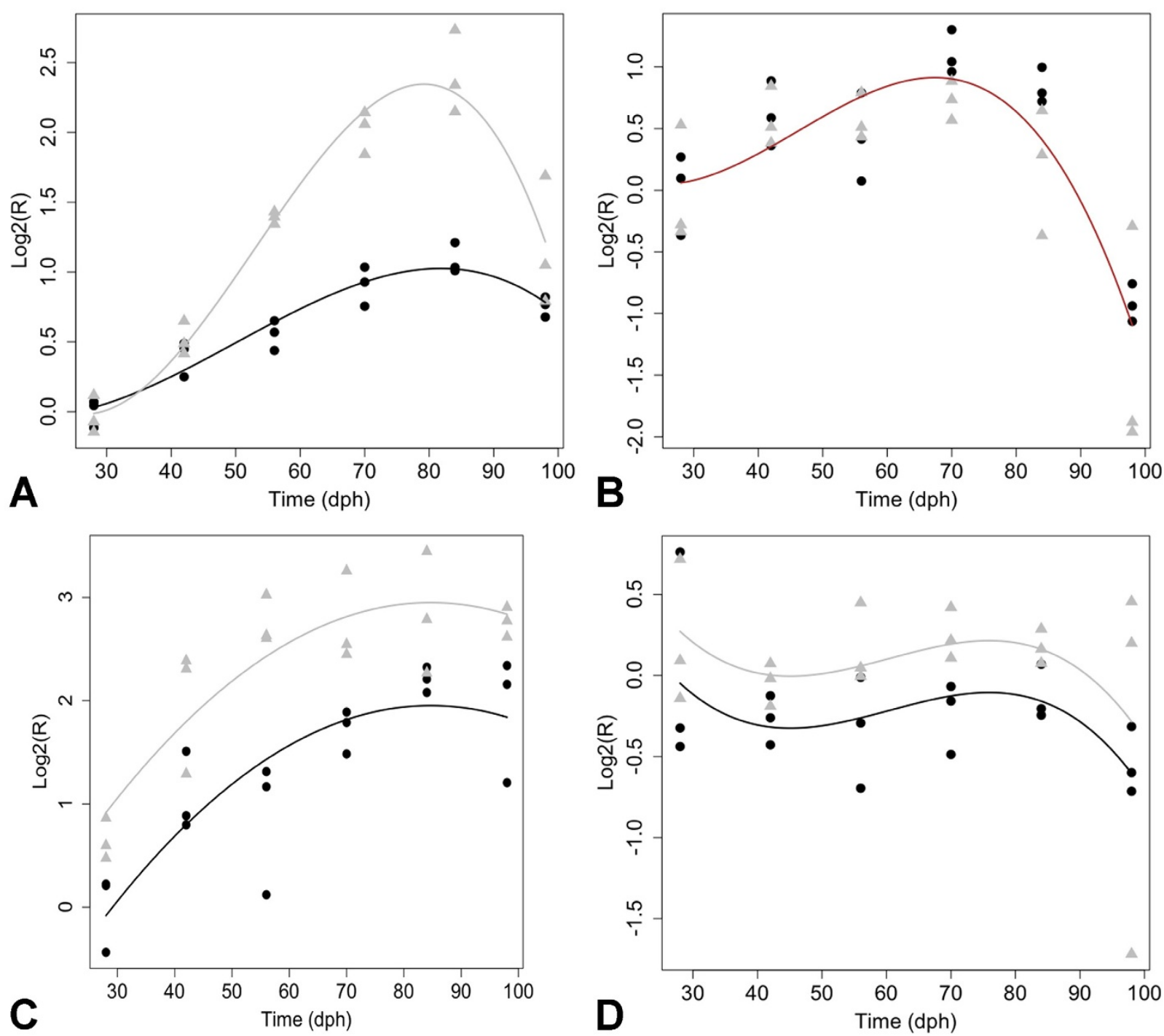

Figure 6 Expression profiles generated by qPCR. The figure shows profiles for hypothalamic-pituitary-interrenal axis genes: (A) $n r 3 c 2$, (B) $n r 3 c 1$, (C) pomc, and (D) crhr1. Axolotl larvae = black circles, tiger salamander larvae = gray triangles. Separate trend lines indicate statistically significant profiles for all genes except $n r 3 c 1$ at the $p=0.05$ level.

during development in both metamorphic and paedomorphic salamanders. Genes associated with vertebrate brain development such as sox3 [32], msx1 [33], and npy [34] significantly increased in abundance in both species. Also $c l u$, a gene expressed at low levels in the central nervous systems of embryonic mice before increasing during postnatal life [35], was similarly up regulated during the axolotl and tiger salamander larval periods. Many aspects of brain development and function are highly conserved among vertebrates. If these functions depend upon conserved patterns of gene transcription, then similarities are expected whether a salamander follows a metamorphic or paedomorphic mode of development.
Although many DEGs were expressed similarly between the species, approximately four times as many were uniquely differentially expressed in tiger salamander larvae. It is possible that many of these gene expression differences represent transcriptional responses in tiger larvae that are necessary for metamorphosis. Three lines of evidence support this idea. (1) More genes were uniquely expressed in tiger larvae and the majority of these showed larger fold changes later in the larval period, when larvae were undergoing anatomical metamorphosis. (2) Hundreds of genes were differentially expressed between axolotls and tiger salamanders throughout larval development, including the earliest time point sampled (42 dph). (3) Genes and gene func- 

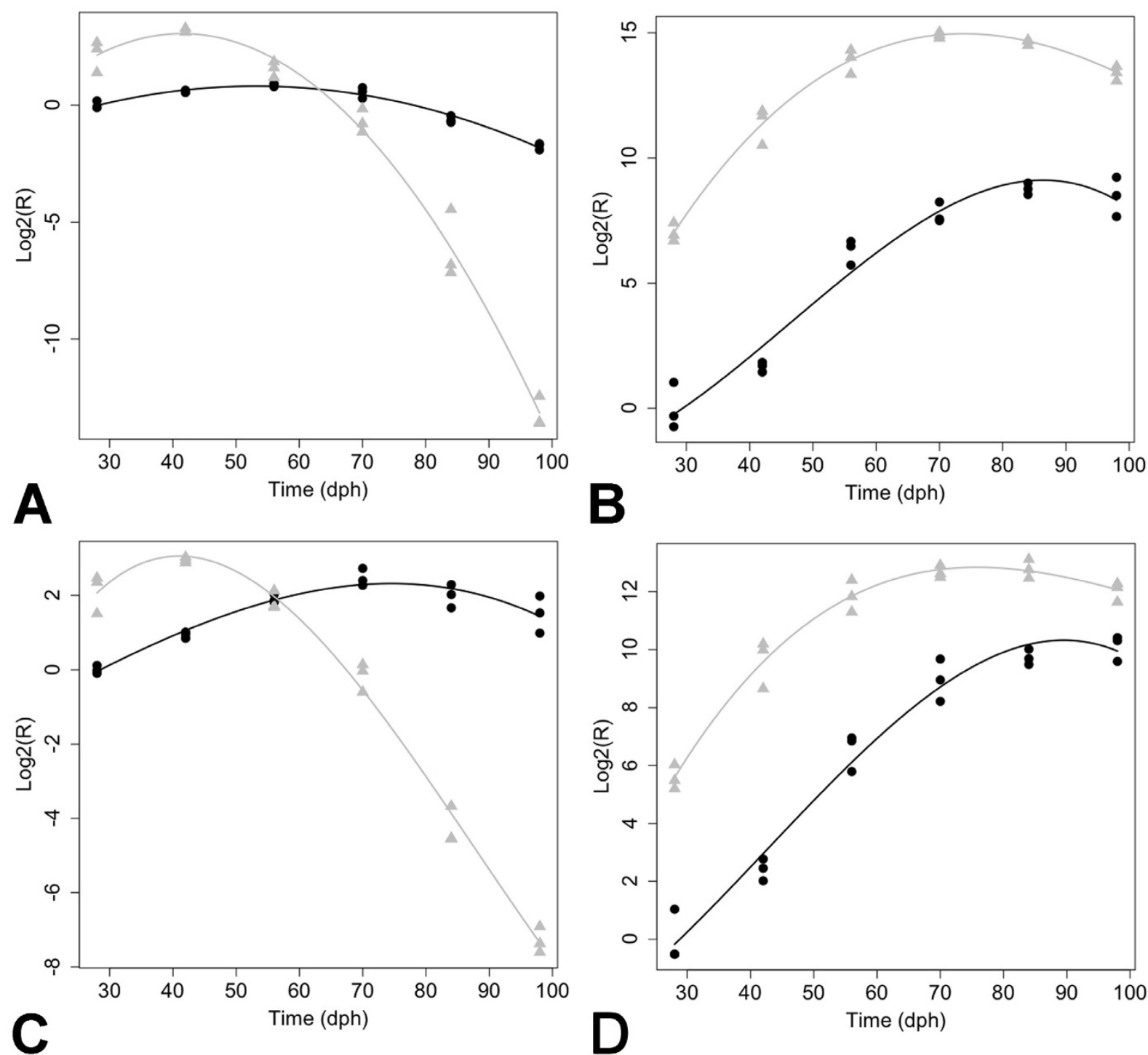

Figure 7 Expression profiles generated by qPCR for hemoglobin genes. (A) hba, (B) hbd, (C) hbel, and (D), hbg 1. Axolotl larvae = black circles, tiger salamander larvae = gray triangles. Separate trend lines indicate statistically significant profiles for all genes at the $p=0.05$ level.

tions that are likely associated with later metamorphic regulation were identified as DEGs. For example, several genes that encode chromatin structure and modifying proteins were uniquely identified from tiger larvae or were differentially expressed between the species (e.g. dnmt1, baz1a, smarca5). Also, several genes that may function in post-transcriptional modification of chromatin proteins were identified as differentially expressed between the species (e.g. sumo1, uba2, ube2e3, ube2I, $u b e 2 l 3, u b e 2 r 2$ ). It is well established that metamorphosis in amphibians and insects requires programming events that activate new transcriptional programs [36]. Indeed, knocking out smt3 (homolog of sumo1) in Drosophila, a gene associated with chromatin remodeling by sumoyla- tion, is known to extend the pupal stage and inhibit metamorphosis [37]. In addition to chromatin-associated genes, the expression of several genes involved in cellular metabolic processes (e.g. cat, got2, adss, acp1, idh3g, ctps, eno3) and hormone pathways (e.g. sstr5, nr3c2, prl) increased or were expressed at higher levels (tef) during tiger salamander development. While it is not unexpected to discover gene expression differences between closely related species, the results are intriguing because transcriptional output was generally higher in tiger salamanders, for the majority of loci that showed differential expression. Moreover, some of the genes that were differentially expressed were identified early in the larval period, well before the onset of morphological metamor- 
phosis. This suggests that metamorphic and paedomorphic modes of development are distinct in a transcriptional sense at very early stages of ontogeny, perhaps tracing back to embryogenesis [16].

Paedomorphosis is a heterochonic term that is classically defined as a change in the timing of development that leads to the retention of ancestral, juvenile characteristics in adults of evolutionarily derived lineages [13]. The simplest model to explain such a pattern is a change that delays the overall rate of development. Patterns of gene expression were discovered that support the idea of developmental delay: axolotls maintained relatively constant $h b e$ and $h b a$ transcript abundances, suggesting the maintenance of an embryonic hemoglobin expression profile throughout larval development. In comparison, transcripts for these genes declined precipitously midway during the larval period in tiger salamanders, perhaps coinciding with the initiation of early, metamorphic changes. However, developmental delay cannot explain all of the axolotl-tiger salamander expression differences. As was noted above, axolotls shared some expression patterns with tiger salamander larvae, which presumably present aspects of the ancestral metamorphic pattern. In addition, axolotls showed unique expression patterns not observed in tiger salamander larvae. Axolotls uniquely up regulated genes that are associated with vertebrate brain development and mammalian brain pathologies, including ctss and aging [38], ogn and pituitary cancer [39], and cd69 and Alzheimers [40]. Also, an expression difference was identified that supports the idea of a depressed HPT axis in axolotls $[21,25]$. Tef, a bZIP transcription factor implicated in the activation of mammalian TSHb [41], showed significantly lower abundances across all time points in axolotl larvae. Finally, consider the transcription of $n r 3 c 1$ and $n r 3 c 2$ during development. The glucocorticoid receptor ( $n r 3 c 1)$ showed a dynamic temporal expression profile that was statistically indistinguishable in both species. Conversely, the mineralocorticoid receptor $(n r 3 c 2)$ was expressed at significantly lower levels in axolotl larvae. Thus, gene expression patterns in larval axolotls appear to be mosaic: some patterns are shared with tiger salamanders and some patterns are novel. Some of the novel patterns may be neutral in effect and fixed by genetic drift. However, in the case of transcription factors that may influence hypothalamic-pituitary development and activity (tef, $n r 3 c 2$ ) or hemoglobins that allow for physiological adaptation to changing oxygen needs, the axolotl provides a model to study expression changes that have likely been selected to better suit large and reproductively competent "larval forms" for a totally aquatic life history.

In considering how discontinuous phenotypes evolve via Darwinian means, Gould [16] proposed an answer in the case of the axolotl: "the problem of reconciling evident discontinuity in macroevolution with Darwinism is largely solved by the observation that small changes early in embryology accumulate through growth to yield profound differences among adults.... Delay the onset of metamorphosis and the axolotl of Lake Xochimilco reproduces as a tadpole with gills and never transforms into a salamander."

Although once a controversial idea, it is accepted now that evolution can act on early stages of development to yield novel phenotypes [42]. There is a lengthy temporal disconnect between embryogenesis and the time a tiger salamander larva first shows morphological changes indicative of metamorphosis (after $56 \mathrm{DPH}$ ). During this time, there is ample time for genetic and environmental factors to affect brain development in ways that alter hypothalamic-pituitary activity late in the larval period. The results show that many genes in the brains of axolotl larvae are transcribed at lower levels than they are in tiger salamander larvae. These include genes that function in the regulation of hypothalamic-pituitary activities that orchestrate anatomical metamorphosis. This suggests the following hypothesis: an axolotl's failure to undergo metamorphosis late in the larval period traces to mechanisms that act early in development to broadly program transcription. This hypothesis can be tested by overexpressing tiger salamander genes in axolotl embryos that function to program gene expression in the brain during early development. Other hypotheses can be tested in axolotls to investigate mechanisms that direct brain development in a predictable manner, towards a hopeful monster outcome.

\section{Conclusion}

This study shows that axolotl and tiger salamander larvae present different brain transcriptional programs and these programs diverge early in development. These early transcriptional differences include genes whose functions associate with a number of biological processes, including cell cycle, apoptosis, chromatin structure and remodeling, cellular metabolism, transcription, posttranslational modification, neural development, and regulation of the HPI and HPT axes. Studies of other metamorphic and paedomorphic species of salamander are needed to disentangle species-specific gene expression responses from those that distinguish metamorphic and paedomorphic modes of development.

\section{Methods}

\section{Study animals}

A single fertilized clutch of $A$. t. tigrinum was obtained from Charles D. Sullivan Co. Inc and the A. mexicanum were sibs deriving from a Voss lab axolotl strain. Larvae from both species were reared individually following hatching in $40 \%$ Holtfretter's solution at $20-22^{\circ} \mathrm{C}$ and fed 
brine shrimp napulii (Artemia sp., Brine Shrimp Direct, Ogden, UT) twice daily for three weeks. After three weeks, larvae were fed California blackworms ad libitum (Lumbriculus sp., J.F. Enterprises, Oakdale, CA). At 28, $42,56,70,84$, and $98 \mathrm{dph}$, salamanders were anesthetized in $0.01 \%$ benzocaine and whole brains and attached pituitaries were flash-frozen in liquid nitrogen immediately following collection. Observations and measurements were collected on larval to monitor the progression of tiger larvae towards metamorphosis. Snout-vent-length (SVL) was recorded for the salamanders from which tissues were collected. A general linear model of the following form was fit to the SVL data: $\mathrm{SVL}_{\mathrm{tij}}=\beta_{0}+\mathrm{S}_{\mathrm{t}}+\mathrm{T}_{\mathrm{i}}+$ $(\mathrm{ST})_{\mathrm{ti}}+\mathrm{T}^{2}{ }_{\mathrm{i}}+\left(\mathrm{ST}^{2}\right)_{\mathrm{ti}}+\varepsilon_{\mathrm{tij}}$ where $\beta_{0}$ corresponds to the intercept term for axolotls, $S_{t}$ corresponds to the intercept term for tiger salamanders, $T_{i}$ corresponds to the linear regression coefficient for axolotls, $(\mathrm{ST})_{\mathrm{ti}}$ corresponds to the linear regression coefficient for tiger salamanders, $\mathrm{T}^{2}{ }_{\mathrm{i}}$ corresponds to the quadratic regression coefficient for axolotls, $\left(\mathrm{ST}^{2}\right)_{\mathrm{ti}}$ corresponds to the quadratic regression coefficient for tiger salamanders, and $\varepsilon_{\mathrm{tij}}$ corresponds to the error term of the jth individual from species $t$ sampled at time i. Animal care and use was approved by the University of Kentucky Animal Care and Use Committee (IACUC protocols \# 01087L2006 and \#00907L2005).

\section{RNA isolation}

Three tissue pools were developed for each time point. Whole brains and attached pituitaries were used because of the small brain size of early larvae, which yielded low amounts of RNA. Each tissue pool contained the brains of three different individuals. Total RNA was isolated using TRIzol (Invitrogen, Carlsbad, CA) and RNA samples were further purified using Qiagen RNeasy mini-columns. RNA samples were quantified via UV spectrophotometry (NanoDrop, ND-1000) and qualified via an Agilent BioAnalyzer (Agilent Technologies).

\section{Gene expression profiling}

Genome-level expression profiling was conducted using a custom Affymetrix GeneChip [28-30]. Three replicate RNA pools for each of four time points $(42,56,70,84$ $\mathrm{dph}$ ) were labeled, hybridized, and scanned by the University of Kentucky Microarray Core Facility according to standard Affymetrix protocols. Additional gene expression profiling for selected genes was conducted for a broader range of time points $(28,42,56,70,84$, and 98 $\mathrm{dph}$ ) using qPCR. Primers (Additional File 5) were designed using Primer3 [43]. When possible, axolotl and tiger salamander orthologs for each gene in Additional File 5 were aligned via BLAST to identify gene regions that corresponded to the same nucleotides covered by
Affymetrix probe-sets. When the orthologs were not $100 \%$ identical in the target regions, separate primers were designed for each species (see Additional File 5). A BioRad iScript Select cDNA synthesis kit (Hercules, CA, USA) was used to synthesize cDNA from $1 \mu \mathrm{g}$ of total RNA and primer efficiencies were estimated separately for axolotl and tiger salamander via linear regression on dilution series. A reference gene (tif1; probe-set L_s_at; Additional File 5) was demonstrated to be invariant across all species by time combinations and relative expression ratios were calculated according to Pfaffl [44]. All expression ratios are relative to the mean expression of axolotl at $28 \mathrm{dph}$ and normalized to tif1. All PCRs were $10 \mu \mathrm{l}$ reactions consisting of $4 \mathrm{ng}$ cDNA, $16.4 \mathrm{ng}$ of forward and reverse primers, and Roche FastStart Universal SYBR Master (Rox) Mix (Roche Diagnostics, Indianapolis, IN). PCRs were conducted on an Applied Biosystems StepOnePlus real-time PCR system. Reaction conditions were as follows: 10 minutes at $95^{\circ} \mathrm{C}, 40$ cycles of $15 \mathrm{sec}$ onds at $95^{\circ} \mathrm{C}$ followed by 1 minute at $55^{\circ} \mathrm{C}, 15$ seconds at $95^{\circ} \mathrm{C}$, and 1 minute at $55^{\circ} \mathrm{C}$. Melting curves were generated to ensure amplification of a single product for each reaction. All reactions were run on 48 well plates and blocked by sampling time and species (i.e., for a given time point, both species were present on the plate). At least two template free controls were present on each plate [45].

\section{Quality control and low-level analyses of the Ambystoma GeneChip}

All arrays were subjected to quality control (QC) at the individual probe level by inspecting box-plots, histograms, pair-wise $M$ versus $A$ plots of replicate GeneChips, pseudo-images of probe level models, and an RNA degradation plot that allows for visualization of the 3' labeling bias across all GeneChips simultaneously [46,47]. Background correction, normalization, and expression summaries were obtained via the robust multi-array average (RMA) algorithm [48]. Two RMA expression matrices were generated separately for each species (see below) and a third RMA expression matrix was computed from all arrays from both species (see below). Upon implementing the RMA algorithm, the probe-set level data from each of these three matrices were subjected to further $\mathrm{QC}$ by inspecting pair-wise $\mathrm{M}$ vs. A plots of replicate GeneChips and examining correlation matrices among replicate GeneChips (minimum mean $r$ for a given species by time point combination across all three of the RMA expression matrices $=0.989$ ). Probe-sets from the two species-specific RMA matrices that were classified as "absent" on > $75 \%$ of the GeneChips were filtered [49]. 


\section{Identification of identical probe-sets}

A total of $1604(\sim 33 \%)$ of the 4844 probe-sets on the Ambystoma GeneChip were designed from contigs that have predicted orthologs in axolotl and tiger salamander. The sequences of the probe-sets were used as queries in BLAST searches of axolotl and tiger salamander EST contigs [50]. BLAST alignments were used to extrapolate the number of mismatches (MM) between microarray probes designed to axolotl and orthologous EST contigs from tiger salamander, and vice versa. These data were then used to calculate the number of probes in each probe-set that had $>0 \mathrm{MM}$ and the sum of MM across each probe-set. Probe-sets that had >0 MM between species were filtered before conducting statistical analyses that directly compared expression values between axolotl and tiger salamander larvae (see below).

\section{Identification of differentially expressed genes}

Two statistical approaches were used to identify DEGs. First, RMA matrices were generated for each species and quadratic regression [51] was used to identify genes that changed as a function of time. This approach also classified genes into nine different temporal profiles based on the values of the estimated regression coefficients (Figure 3 ; see also [52]). The "flat" profile describes genes that do not show transcript abundance changes (null results). The LU, LD, QLVU, and QLCD profiles described genes that show linear (LU, LD) or nonlinear (QLVU, QLCU, QLCD, QLVD) changes in transcript abundance across sample times. The QV and QC expression profiles described genes that show transient changes. Statistical correction for multiple testing was done separately for each species by evaluating $\alpha_{0}$ at a false discovery rate (FDR- [53]) of 0.05. $\alpha_{1}$ was set to 0.05 . In addition to statistical criteria, genes were only retained if they exhibited $\geq 1.5$ fold changes relative to $42 \mathrm{dph}$ (baseline) at one or more of the other time points (56,72, or $84 \mathrm{dph}$ ).

The second statistical approach used the global RMA matrix to directly compare axolotl and tiger salamander expression levels/profiles for genes known to exhibit zero sequence divergence in the regions encompassed by Affymetrix probe-sets (see above). This analysis was conducted using the maSigPro software package [54] that is available from bioconductor http://www.bioconductor.org for the R statistical computing environment http:/ /www.r-project.org. In short, maSigPro was used to fit second order (i.e., quadratic) regression models, in which the species term is identified by a dummy variable, in a gene-by-gene manner. Correction for multiple testing was achieved by evaluating the over-all model P-values according to the algorithm of Benjamini and Hochberg [53] at an FDR of 0.05. A backward selection procedure was then used to eliminate non-significant $(\alpha=0.05)$ terms from significant models. In order for genes from this analysis to be considered "identified" they had to meet the following criteria: (1) over-all model $P$-values lower than the FDR adjusted threshold, (2) significant species, time $\times$ species, or time ${ }^{2} \times$ species terms, (3) an $R^{2}$ $\geq 0.50$, and (4) a $\geq 1.5$ fold difference between axolotl and tiger salamander at one or more of the sampling times $(42,56,70$, or $84 \mathrm{dph})$.

\section{Identification of statistically enriched biological processes}

To identify biological processes that were statistically enriched in our lists of DEGs, we conducted EASE analyses using the database for annotation visualization and integrated discovery (DAVID)[55]. For all analyses, the 3728 genes on the Ambystoma GeneChip with established orthologies to humans were used to generate expected values (i.e., as the background). The count threshold was always set to two and the EASE threshold was always set to 0.05 . The list of significant GO terms was manually inspected to remove redundant terms.

\section{Statistical analysis of the qPCR data}

General linear models were fit to qPCR estimates of mRNA abundance to determine if gene expression differed in magnitude and/or temporal profile between the species. These models took the form: $\log _{2}(R)_{\mathrm{tij}}=\beta_{0}+\mathrm{S}_{\mathrm{t}}+$ $\mathrm{T}_{\mathrm{i}}+(\mathrm{ST})_{\mathrm{ti}}+\mathrm{T}^{2}{ }_{\mathrm{i}}+\left(\mathrm{ST}^{2}\right)_{\mathrm{ti}}+\mathrm{T}^{3}{ }_{\mathrm{i}}+\left(\mathrm{ST}^{3}\right)_{\mathrm{ti}}+\varepsilon_{\mathrm{tij}}$ where $\beta_{0}=$ the intercept term for axolotl, $S_{t}=$ the intercept term for tiger salamander, $T_{i}=$ the linear regression coefficient for axolotl, $(\mathrm{ST})_{\mathrm{ti}}=$ an additional linear regression coefficient for tiger salamander, $\mathrm{T}^{2}{ }_{\mathrm{i}}=$ the quadratic regression coefficient for axolotl, $\left(\mathrm{ST}^{2}\right)_{\mathrm{ti}}=$ an additional quadratic regression coefficient for tiger salamander, $\mathrm{T}^{3}{ }_{\mathrm{i}}=$ the trinomial regression coefficient for axolotl, $\left(\mathrm{ST}^{3}\right)_{\mathrm{ti}}=$ an additional trinomial regression coefficient for tiger salamander, and $\varepsilon_{\mathrm{tij}}=$ the error term associated with jth RNA pool from species $t$ and time $i$. When necessary, these models were simplified via a backward selection scheme that removed non-significant terms $(P>0.05)$.

\section{Additional material}

Additional file 1 Differentially expressed genes identified from axolotl and tiger salamander larvae. Table contains 134 total probesets that correspond to 108 different genes identified from larval axolotls and tiger salamanders. BLASTx searches were performed to identify presumptive human orthologs. The regression profile for each gene is presented. Additional file $\mathbf{2}$ Genes uniquely identified from axolotl larvae. Table contains 85 total probesets that correspond to 76 different genes identified from larval axolotls. BLASTx searches were performed to identify presumptive human orthologs. The regression profile for each gene is presented. Additional file 3 Genes uniquely identified from tiger salamander larvae. Table contains 332 probesets that correspond to 292 different genes identified from a direct comparison of gene expression between the brains of larval axolotls and tiger salamanders. BLASTx searches were performed to identify presumptive human orthologs. The regression profile for each gene is presented. 


\section{Additional file 4 Differentially expressed genes identified from axo-} lotl and tiger salamander larvae. Table contains 419 probesets that correspond to 409 different genes identified from a direct comparison of gene expression between the brains of larval axolotls and tiger salamanders. BLASTx searches were performed to identify presumptive human orthologs.

\section{Additional file 5 Primer sequences used for qPCR assays. Table of} genes and corresponding primer sequences that were used to accomplish qPCR. For the "Species" column, "Both" denotes genes with identical sequences between A. mexicanum and A. t. tigrinum, "Tiger Only" denotes genes for which sequence information was only available from A. t. tigrinum "Axolotl Only" denotes genes for which sequence information was only available from A. mexicanum, "Tiger" denotes primers that were designed from A. t. tigrinum sequence, and "Axolotl" denotes primers that were designed from $A$. mexicanum sequence. + /- denotes the direction of a given primer

\section{Authors' contributions}

RBP designed the microarray experiment, conducted statistical and bioinformatics analyses, analyzed data and interpreted results, and contributed to the writing of the manuscript. MAB collected morphometric measurements and tissue samples from A. mexicanum, isolated RNA and performed qPCR, analyzed and interpreted data, and contributed to the writing of the manuscript. JJS conceived the temporal sampling design and collected morphometric measurements and tissue samples from A. t. tigrinum. SP helped with bioinformatic analyses. SRV analyzed data and interpreted results, and led the manuscript writing effort. All authors have read and approved the final manuscript.

\section{Acknowledgements}

We thank James Monaghan, John Walker, Phil Crowley, and Bruce O'Hara for helpful discussion and comments. Donna Walls and the University of Kentucky Microarray Core Facility processed samples for array analysis. The research was supported by grant R24-RR016344 from the National Center for Research Resources (NCRR), a component of the National Institutes of Health (NIH). Its contents are solely the responsibility of the authors and do not necessarily represent the official views of NCRR or $\mathrm{NIH}$. The project was also supported by funds from the Kentucky Spinal Cord and Brain Injury Research Trust. The Spinal Cord and Brain Injury Research Center and the NSF supported Ambystoma Genetic Stock Center (DBI-0443496) provided resources and facilities.

\section{Author Details}

'Department of Biology and Spinal Cord and Brain Injury Research Center, University of Kentucky, Lexington, KY 40506, USA and 2Benaroya Research Institute, Seattle, WA 98101, USA

Received: 16 March 2010 Accepted: 28 June 2010

Published: 28 June 2010

\section{References}

1. Darwin C: On the Origin of Species by Means of Natural Selection, or the Preservation of Favoured Races in the Struggle for Life. John Murray, London; 1859

2. Goldschmidt R: The Material Basis of Evolution. Yale University Press. New Haven; 1940.

3. Simpson GG: Tempo and Mode in Evolution. Columbia University Press, New York: 1940

4. Hoekstra HE, Hirschmann RJ, Bundey RA, Insel PA, Crossland JP: A single amino acid mutation contributes to adaptive beach mouse color pattern. Science 2006, 313:101-104.

5. Cresko WA, Amores A, Wilson C, Murphy J, Currey M, Phillips P, Bell MA Kimmel CB, Postlethwait JH: Parallel genetic basis for repeated evolution of armor loss in Alaskan threespine stickleback populations. Proc Natl Acad Sci 2004, 101:6050-6065.

6. Schemske DW, Bradshaw HD Jr: Pollinator preference and the evolution of floral traits in monkeyflowers (Mimulus). Proc Natl Acad Sci 1999. 96:11910-11915.

7. Doebley JA, Stec A, Hubbard L: The evolution of apical dominance in maize. Nature 1997, 386:485-488.
8. Voss SR, Shaffer HB: Adaptive evolution via a major gene effect: paedomorphosis in the Mexican axolotl. Proc Natl Acad Sci 1997 94:14185-14189.

9. Theissen $\mathrm{G}$ : The proper place of hopeful monsters in evolutionary biology. Theory Biosci 2006, 124:349-369.

10. Theissen G: Saltational evolution: hopeful monsters are here to stay. Theory Biosci 2009, 128:43-51.

11. Shaffer HB, Voss SR: Phylogenetic and mechanistic analysis of a developmentally integrated character complex: Alternate life history modes in ambystomatid salamanders. Am Zool 1996, 36:24-35.

12. Kollman J: Das Ueberwintern von europäis-chen Frosch- und Tritonlarven und die Umwand-lung des mexikanischen Axolotl. Verhandlungen der Naturforschenden Gesellschaft in Basel 1885, 7:387-398.

13. Gould SJ: Ontogeny and Phylogeny. Belkap Press, Cambridge, MA; 1977.

14. Huxley JS: Metamorphosis of axolotl caused by thyroid feeding. Nature 1920, 104:2618.

15. Gadow H: The Mexican axolotl. Nature 1903, 67:330-332

16. Gould SJ: The Return of Hopeful Monsters. Nat Hist 1977, 86:22-30.

17. Voss SR: Genetic basis of paedomorphosis in the axolotl, Ambystoma mexicanum: a test of the single gene hypothesis. J Hered 1995, 86:441-447.

18. Voss SR, Shaffer HB: Evolutionary genetics of metamorphic failure using wild-caught vs. laboratory axolotls (Ambystoma mexicanum). Mol Ecol 2000, 9:1401-1407.

19. Voss SR, Prudic K, Oliver J, Shaffer HB: Candidate gene analysis of metamorphic timing in ambystomatid salamanders. Mol Ecol 2003, 12:1217-1223

20. Voss SR, Smith JJ: Evolution of salamander life cycles: A major-effect quantitative trait locus contributes to discrete and continuous variation for metamorphic timing. Genetics 2005, 170:275-281.

21. Ducibella T: The occurrence of biochemical metamorphic events without anatomical metamorphosis in the axolotl. Dev Biol 1974, 38:175-86.

22. Galton VA: Thyroid hormone receptors and iodothyronine deiodinases in the developing Mexican axolotl, Ambystoma mexicanum. Gen Comp Endo 1991, 85:62-70.

23. Rosenkilde $P$ : The role of hormones in the regulation of amphibian metamorphosis. In Metamorphosis Edited by: Balls M, Bownes M. Oxford: Clarendon Press; 1985:221-259.

24. Kuhn ER, Jacobs GFM: Metamorphosis. In Developmental Biology of the Axolot/ Edited by: Armstrong J, Malacinski G. Oxford, Oxford University Press: 1989:187-197.

25. Rosenkilde P, Ussing AP: What mechanisms control neoteny and regulate induced metamorphosis in urodeles? Int J Dev Biol 1996, 40:665-673

26. Tompkins R: Genic control of axolotl metamorphosis. Amer Zool 1978, 18:313-319.

27. Gould SJ: Change in developmental timing as a mechanism of macroevolution. In Evolution and Development Edited by: Bonner JT. Berlin, Springer-Verlag; 1981:333-346

28. Monaghan JR, Walker JA, Page RB, Putta S, Beachy CK, Voss SR: Early gene expression during natural spinal cord regeneration in the salamanders Ambystoma mexicanum. J Neurochem 2007, 101:27-40.

29. Page RB, Monaghan JR, Samuels AK, Smith JJ, Beachy CK, Voss SR: Microarray analysis identifies keratin loci as sensitive biomarkers for thyroid hormone disruption in salamanders (Ambystoma). Comp Bioch Physiol Part C 2007, 145:15-27.

30. Page RB, Monaghan JR, Walker JA, Voss SR: A model of transcriptional and morphological changes during thyroid hormone-induced metamorphosis of the axolotl. Gen Comp Endo 2009, 162:219-32.

31. Rifkin SA, Kim J, White KP: Evolution of gene expression in the Drosophila melanogaster subgroup. Nat Genet 2003, 33:138-44.

32. Nikcevi( G, Savi( T, Kovacevi(-Grujici( N, Stevanovi( M: Up-regulation of the SOX3 gene expression by retinoic acid: characterization of the novel promoter-response element and the retinoid receptors involved. J Neurochem 2008, 107:1206-15.

33. Bach A, Lallemand Y, Nicola MA, Ramos C, Mathis L, Maufras M, Robert B. Msx1 is required for dorsal diencephalons patterning. Development 2003, 130:4025-4036.

34. Agasse F, Bernardino L, Kristiansen H, Christiansen SH, Ferreira R, Silva B, Grade S, Woldbye DP, Malva JO: Neuropeptide Y promotes 
neurogenesis in murine subventricular zone. Stem Cells 2008, 26:16636-1645.

35. Charnay Y, Imhof A, Vallet PG, Hakkoum D, Lathuiliere A, Poku N, Aronow B, Kovari E, Bouras C, Giannakopoulos P: Clusterin expression during fetal and postnatal CNS development in mouse. Neuroscience 2008, 155:714-724.

36. Tata JR: Gene expression during metamorphosis: an ideal model for post-embryonic development. Bioessays 1993, 15:239-48.

37. Talamillo A, Sánchez J, Cantera R, Pérez C, Martín D, Caminero E, Barrio R: $\mathrm{Smt} 3$ is required for Drosophila melanogaster metamorphosis. Development 2008, 135:1659-1668

38. Wendt W, Lübbert H, Stichel CC: Upregulation of cathepsin S in the aging and pathological nervous system of mice. Brain Res 2008, 1232:7-20.

39. Hu SM, Li F, Yu HM, Li RY, Ma QY, Ye TJ, Lu ZY, Chen JL, Song HD: The mimecan gene expressed in human pituitary and regulated by pituitary transcription factor- 1 as a marker for diagnosing pituitary tumors. J Clin Endo Met 2005, 90:6657-6664.

40. Kusdra L, Rempel H, Yaffe K, Pulliam L: Elevation of CD69+ monocyte/ macrophages in patients with Alzheimer's disease. Immunnobiology 2000, 202:26-33

41. Drolet DW, Scully KM, Simmons DM, Wegner M, Chu KT, Swanson LW, Rosenfeld MG: TEF, a transcription factor expressed specifically in the anterior pituitary during embryogenesis, defines a new class of leucine zipper proteins. Genes Dev 1991, 5:1739-1753.

42. Raff RA: The Shape of Life. Genes, Development, and the Evolution of Animal Form. University Chicago Press, Chicago; 1996

43. Rozen S, Skaletsky H: Primer3 on the www for general users and for biologist programmers. Methods Mol Biol 2000, 132:365-86.

44. Pfaffl M: A new mathematical model for relative quantification in realtime RT-PCR. Nucleic Acids Research 2001, 29:e45.

45. Bustin SA, Nolan T: Pitfalls of quantitative real-time reversetranscription polymerase chain reaction. J Biomolec Techniques 2004, 15:155-66.

46. Bolstad BM, Irizarry RA, Gautier L, Wu Z: Preprocessing high-density oligonucleotide arrays. In Bioinformatics and Computational Biology Solutions Using R and Bioconductor Edited by: Gentaleman R, Carey VJ, Huber W, Irizarry RA, Dudoit S. New York: Springer; 2005:13-32.

47. Bolstad BM, Collin F, Brettschneider J, Simpson K, Cope L, Irizarry RA, Speed TP: Quality assessment of Affymetrix GeneChip data. In Bioinformatics and Computational Biology Solutions Using R and Bioconductor Edited by: Gentaleman R, Carey VJ, Huber W, Irizarry RA, Dudoit S. New York: Springer; 2005:33-47.

48. Izarry RA, Hobbs B, Collin F, Beazer-Barclay YD, Antonellis KJ, Scherf U, Speed TP: Exploration, normalization, and summaries of high density oligonucleotide array probe level data. Biostatistics 2003, 4:249-264.

49. McClintick JN, Edenberg HJ: Effects of filtering by present call on analysis of microarray experiments. BMC Bioinformatics 2006, 7:49

50. Smith JJ, Putta S, Walker JA, Kump DC, Samuels AK, Monaghan JR, Weisrock DW, Staben C, Voss SR: Integrating new and existing ambystomatid research and information resources. BMC Genomics 2005, 6:181

51. Liu H, Tarima S, Borders AS, Getchell TV, Gertchell ML, Stromberg AJ: Quadratic regression analysis for gene discovery and pattern recognition for non-cyclic short time-course microarray experiments. BMC Bioinformatics 2005, 6:106-123.

52. Page RB, Voss SR, Samuels AK, Smith JJ, Putta S, Beachy CK: Effect of thyroid hormone concentration on the transcriptional response underlying induced metamorphosis in the Mexican axolot (Ambystoma). BMC Genomics 2008, 9:78

53. Benjamini $Y$, Hochberg $Y$ : Controlling the false discovery rate: a practical and powerful approach to multiple testing. J Royal Stat Soc, B 1995, 57:289-300

54. Conesa A, Nueda MJ, Ferrer A, Talon M: maSigPro: a method to indentify significantly differential expression profiles in time-course microarray experiments. Bioinformatics 2006, 22:1096-1102.

55. Dennis G, Sherman BT, Hosack DA, Yang J, Gao W, Lane HC, Lempicki RA: DAVID: database for annotation, visualization, and integrated discovery. Genome Biology 2003, 4:R60. doi: 10.1186/1471-2148-10-199

Cite this article as: Page et al., Microarray analysis of a salamander hopeful monster reveals transcriptional signatures of paedomorphic brain development BMC Evolutionary Biology 2010, 10:199

\section{Submit your next manuscript to BioMed Central and take full advantage of:}

- Convenient online submission

- Thorough peer review

- No space constraints or color figure charges

- Immediate publication on acceptance

- Inclusion in PubMed, CAS, Scopus and Google Scholar

- Research which is freely available for redistribution
C Biomed Central 